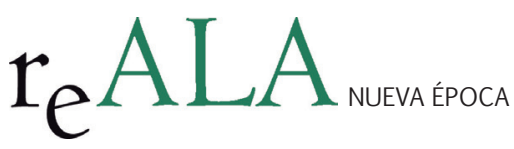

REALA, n 2, julio-diciembre 2014, ISSN: 1989-8975

DOI: http://dx.doi.org/10.24965/reala.voi2.10191

\title{
Estado de Derecho, Estado Autonómico y Energías Renovables
}

en Italia

\author{
Giuseppe Franco Ferrari \\ Catedrático de Derecho Público comparado. Universidad Bocconi de Milán \\ Ferrari.giuseppe@unibocconi.it \\ Recibido: 25 de enero 2014 \\ Aceptado: 2 febrero 2014
}

\section{Resumen}

Este artículo describe la evolución de la situación normativa y factual de las energías renovables en el ordenamiento italiano - La dependencia energética del extranjero debido a falta de materias primas clásicas ha influido sobre el desarrollo del sector hídrico, del geotérmico y de las bioenergías, mientras que el eólico y el fotovoltaico han encontrado obstáculos en la sensibilidad de las poblaciones locales hacia las temáticas del medio-ambiente - El sector de la energía atómica conoció un desarrollo vivaz y precoz hasta la mitad de los años '80, cuando varios referéndums populares abrogaron las normas concernientes localización y construcción de las centrales. Desde entonces el ordenamiento italiano solo se ha ocupado del desmantelamiento de las plantas existentes.

Palabras clave

Energías renovables, nuclear, eólica, fotovoltaico, bioenergías, geotérmicas

\section{Rule of Law, State Autonomy and Renewable Energy in Italy}

Abstract

This article describes the evolution of the Italian legal and factual framework concerning renewable energies. The strong dependence on foreign sources, caused by the lack of carbon and petrol has influenced the development of the hydric sector, of geothermical energies and bionergies. Wind and photovoltaic energies have been obstacled by the resistance of the local population towards possible damages to the environment. At the same time, the use of atomic energy was precociously developed in the '60s and '70s, till 1987. At that time, three referenda abrogated the most important provisions concerning localization and construction of atomic plants. Since then, the Italian legal system has dedicated many efforts for the dismantling of existing plants.

\section{Keywords}

Renewables, nuclear, wind, photovoltaic, bioenergy, geothermal 


\section{SUMARIO}

\section{Introducción; 2. Energía hidroeléctrica; 3. Energía nuclear; 4. Energía eólica: 5. Sector fotovoltaico; 6. Bioenergías; 7. Energías geotérmicas.}

\section{INTRODUCCIÓN}

Las energías renovables en el ordenamiento italiano presentan aspectos muy importantes desde el punto de vista constitucional, posiblemente debido a factores domésticos peculiares, de naturaleza tanto institucional cuanto política. Algunos problemas, concernientes a la repartición de competencias entre Estado y Regiones de autonomía especial, en cuyo territorio se encontraban muchas fuentes de energía hidroeléctrica, fueron resueltos en los primeros años de la vida de la República, entre 1946 y 1948, aunque no han acabado nunca de fomentar tensiones, en primer lugar por su relevante valor económico; otros, en punto a la energía atómica, con tal de que pueda considerarse renovable y no solamente alternativa, habían sido afrontados con bastante antelación en comparación con otros países europeos, pero al final se bloquearon en los años 80, por causa de la oposición de movimientos y partidos verdes y radicales, y por fin de una amplia mayoría de la opinión pública; otros, en el sector de la energía eólica, surgieron bastante tarde y también acabaron bloqueados en la última década también por el conflicto entre la falta de energía barata y el deseo de protección del territorio y del paisaje; por fin, las técnicas fotovoltaicas han suscitado también problemas de protección del paisaje, después de haber llegado a una discreta difusión, sobre todo en las Regiones del Sur.

El conjunto de esos factores ha generado una dependencia de las fuentes energéticas tradicionales que sobrepasa la de todos los principales países del occidente ${ }^{1}$ y ha influido muchísimo sobre el costo de las materias primas, poniendo el sistema industrial italiano en una posición de desventaja frente a los otros sistemas industriales, capaces de producir mayores cantidades de energía a precios más bajos. Sin embargo, en 2012, de un total de $340.400 \mathrm{GWh}$ consumidos, un $27,1 \%$ han sido suministrados por fuentes renovables, con una alza del $3 \%$ en comparación con el año anterior. El reparto interno es el siguiente: $12,3 \%$ depende del sector hídrico, $1,6 \%$ del geotérmico, 3,9 del eólico (2,8 en 2011, con un incremento del 36\%), 5,5\% del fotovoltaico (3,1 en 2011 , con un incremento del 74,7\% en el año), 3,7\% de bioenergías (3,1 en 2011, con acrecimiento del 15,3\% en el año).

El texto constitucional original no hacía ninguna mención de la materia energía, y tampoco de las energías renovables. En consecuencia, el sector pertenecía sin duda a la competencia del Estado. La revisión constitucional del Título V en 2001 introdujo en las competencias legislativas concurrentes "producción, transporte y distribución nacional de energía”, asignando así los principios a la ley estatal y su ejecución en detalle a las Regiones. Por lo tanto, hasta 2001 los problemas de reconstrucción de la materia se resolvían según los criterios interpretativos constitucionales generales, por lo menos fuera de las Regiones especiales, cuyos estatutos autonómicos ya contenían disposiciones específicas; después de 2001, en cambio, muchas cuestiones interpretativas tuvieron que ser encaradas desde la perspectiva de la división de competencias entre Estado y Regiones, tanto en casos de impugnación principal de leyes regionales por el Estado o estatales por las Regiones, como en conflictos de atribución referidos a actos administrativos, y también en casos de devolución incidental a la Corte constitucional.

\section{ENERGÍA HIDROELÉCTRICA}

La presencia de una parte montañosa muy considerable del territorio nacional ha representado para Italia una relevante fuente de riqueza en forma de energía hidroeléctrica, tanto en las Regiones alpinas cuanto en la dorsal apenina. De hecho en 1933, cuando fué adoptado el Texto único de las aguas, con real decreto $1775^{2}$, un porcentaje muy alto del total de la producción italiana de energía eléctrica era imputable a derivaciones de aguas públicas y centrales hidroeléctricas realizadas por empresas privadas en régimen de concesión o por haciendas municipales, como la Azienda elettrica municipale (AEM) de Milán, que desde el final del siglo XIX construyó varios diques y establecimientos de producción de energía eléctrica en el norte de Lombardía para proveer de electricidad a la ciudad metropolitana y a los municipios limítrofes. Entre 1933 y 1950 prácticamente no había ${ }^{3}$ otras fuentes de energía que la hidroeléctrica. La construcción de plantas alimentadas con combustibles a base de petróleo y sus derivados empieza con la nacionalización de 1962 y con las masivas inversiones de ENEL. De hecho, en 1933 la producción hidroeléctrica era de $11.300 \mathrm{GWh}$, la termoeléctrica tradicional de 274 , y nunca había sobrepasado el nivel de 350; en 1951, restablecida la producción industrial después de los daños de la guerra mundial, la primera

1 Italia, según datos Enel de 2011, tiene la mayor dependencia energética del extranjero, antes que España, y la mayor dependencia de las fuentes tradicionales.

2 R.D.11 diciembre 1933, n.1775

3 Datos Terna.

REALA, n² 2 julio-diciembre 2014, ISSN: 1989-8975 - DOI: http://dx.doi.org/10.24965/reala.v0i2.10191 
llegaba a $26.354 \mathrm{GWh}$, la segunda a 1.284; desde entonces la primera sigue creciendo con regularidad, la segunda mucho más rápidamente, con el crecimiento impetuoso de la demanda doméstica e industrial en los años 50 y 60, hasta que en 1967 la producción termoeléctrica adelanta a la hidroeléctrica, en 1972 sube al doble, en 1976 al triple, en 1989 al cuádruple, también debido a la desaparición de la nucleotermoeléctrica en 19874. La producción de energía de origen hídrico puede por su naturaleza subir sólo lentamente, y encontrando el límite insuperable de la razonable explotación de los recursos naturales. Por eso, de los 46.107 GWh de 1963 sube a los 54.407 de 2010, nivel máximo de siempre.

Entre otras cosas, en el nuevo siglo Regiones y administraciones locales empiezan a obstaculizar con fuerza nuevas solicitudes de desviaciones hídricas con la finalidad de producción eléctrica, en gran parte por razones de protección del medio ambiente frente al consumo y a la explotación del territorio. La oposición se manifiesta con la creciente complicación de los procedimientos administrativos, no tanto para la concesión, como para los permisos de construcción de las obras civiles necesarias de competencia municipal, una vez conseguida la concesión, o incluso, por ejemplo, en la expresión de opinión negativa en la conferencia de servicios que precede a la emanación de la concesión. El contencioso en los tribunales administrativos regionales y en el Consejo de Estado crece de hecho exponencialmente ${ }^{5}$ n no es raro que acabe con la anulación de actos de denegación de permisos e indemnización de perjuicios ocasionados por el retraso.

El texto único de 1933, en tiempos de centralización, había asignado al Estado y a sus órganos periféricos la competencia en la gestión del patrimonio hídrico. La progresiva actuación del regionalismo después de 1970 ha comportado la devolución a las Regiones ordinarias de muchas tareas en cuanto a la titularidad y al uso de las aguas. Empezaron, antes de la revisión constitucional de 2001, la ley 36/1994 ${ }^{6}$ y los decretos legislativos 112/1998 y 79/19997, cuyo impacto en conjunto ha sido la devolución de casi todas las competencias estatales a las Regiones ordinarias.

En cuanto a las Regiones especiales, sus historias son bastante diferentes. En Valle d'Aosta, la versión originaria del Estatuto ${ }^{8}$ daba a la Región competencia exclusiva en materia de aguas públicas para riego y uso doméstico (art. 2, letr. m) y confería una competencia de integración y de actuación en materia de disciplina de uso de aguas públicas para uso hidroeléctrico (art. 3, letr.d). Las aguas públicas no concesionadas todavía pasaban a la Región a título de concesión por 99 años, pudiendo formar objeto de sub-concesión (art. 7), de acuerdo con una contribución anual o tarifa no superior a la suma determinada por el Estado, con opinión de la Junta regional. Tres entidades privadas desde el principio del siglo XX habían comenzado a construir centrales: SIP, Edison y Ansaldo. En 1933 IRI adquirió la propiedad de SIP. En 1962 la Región impugnó la nacionalización eléctrica, pero sin éxito9. Entre 1991 y 2001 la Región compró muchas de las centrales existentes en el territorio regional al través de una sociedad anónima (Finaosta), y luego creó la Compagnia Valdostana delle acque (CVA), controlada por Finaosta. La Región obtiene beneficios superiores a los 7 millones al año de las contribuciones de concesiones y sub-concesiones. La Región ha tratado de introducirse en el segmento de la venta, además de la distribución, a través de Vallenergie, y por ley ha introducido políticas tarifarias propias, por ejemplo reembolsando tarifas a los residentes por sus primeras viviendas y pactando con algunos concesionarios para lograr descuentos. La Autoridad Antitrust ha contrastado eficazmente estos intentos por tratarse de conductas anti-competenciales.

En Cerdeña el Estatuto de $1948^{10}$ ha asignado a la Región competencia exclusiva en tema de ejercicio de los derechos de la empresa pública de aguas. En Sicilia, el Estatuto" confía a la ley regional la regulación de obras públicas y aguas públicas, siempre y cuando no estén afectadas por obras públicas de interés nacional. En Friuli Venezia-Giulia el Estatuto de $1963^{12}$ deja a la Región el uso de las aguas públicas, con exclusión de las grandes derivaciones, con formulación muy parecida a la del Trentino-Alto-Adigio, en el texto estatutario modificado en $1972^{13}$.

Se ha suscitado mucha conflictividad a lo largo de los años, tanto antes como después de la revisión constitucional de 2001, sobre varios asuntos. Una de las temáticas más frecuentemente discutidas ha sido la de la medida de las contribuciones anuales de los concesionarios, ya que las Regiones especiales a veces han intentado

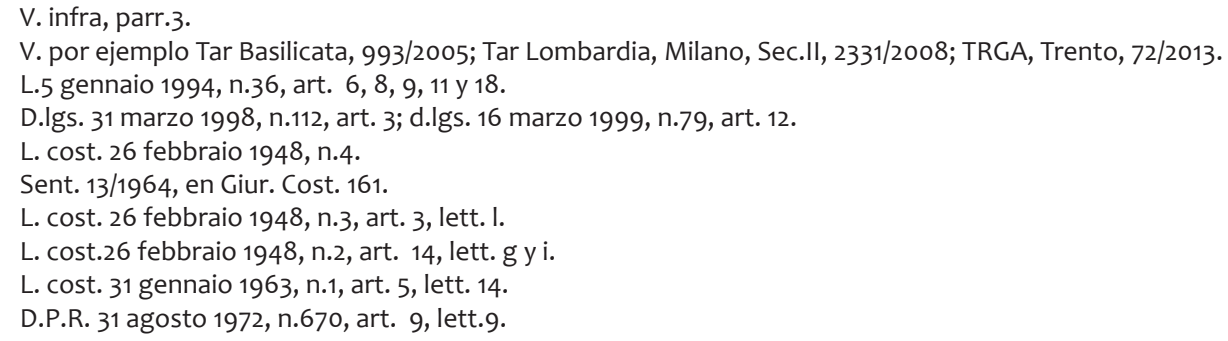


introducir criterios más remuneradores que los estatales, vetados por entrar en contraste con los principios de racionalidad y proporcionalidad. Cuestiones incidentales de constitucionalidad han sido promovidas por el Tribunal superior de aguas públicas y por la Corte de casación ${ }^{14}$. Varias veces la Corte constitucional tuvo que pronunciarse sobre la diferenciación progresiva y no proporcional de las contribuciones entre categorías de derivaciones (grandes o pequeñas) o hasta en la misma según la capacidad'5, con respeto a leyes tanto de Regiones especiales como ordinarias $^{16}$. Considerada la importancia económica de la energía hidroeléctrica, leyes de este tipo han sido vetadas también por vulneración de la competencia exclusiva estatal en materia de Derecho de la competencia (art. 117, aptd.2, let. e), y la Corte se ha adherido ${ }^{17}$, y en la misma perspectiva ha declarado inconstitucional la prórroga automática de concesiones llegadas a vencimiento ${ }^{18}$. Hay también que tener en cuenta, para explicar el rigor de la Corte, que más del $90 \%$ de los recursos hídricos nacionales han sido utilizados y que el mercado está prácticamente cerrado.

\section{ENERGÍA NUCLEAR}

De la energía atómica el legislador italiano empezó a ocuparse, como he dicho, muy temprano. La ley de nacionalización de la energía eléctrica de $1962^{19}$, que creó el ENEL (Ente nacional para la energía eléctrica) bajo vigilancia del Ministerio de Hacienda y según directivas de un Comité ministerial, le hizo responsable de cualquier forma de producción, importación y exportación, transporte, transformación, distribución y venta de energía eléctrica producida por cualquier fuente, incluyendo todas las clases de energía que el desarrollo tecnológico hiciera factibles. Casi contemporáneamente, otra ley de $1962^{20}$ reguló el empleo pacífico de la energía nuclear. El artículo único de la ley 856/1973 ${ }^{21}$ insertó en la de 1962, art. 1, aptd. 5, una letra b, que expresamente mencionaba entre los objetivos de ENEL la realización y la puesta en funcionamiento de plantas nucleares. En los años ' 60 y '70, con buena capacidad de observar el paso del progreso tecnológico y de la extraordinaria expansión industrial del país, ENEL proyectó, realizó y llegó hasta el punto de poner en funcionamiento cuatro plantas ${ }^{22}$ con una capacidad total de $3.510 \mathrm{MW}$ en 1965, que llegaron a 4.428 en 1978, a 7.024 en 1985 y a 8.758 en $1986^{23}$. El procedimiento de localización de las centrales fue disciplinado en 1975². Desde 1952 el Gobierno de De Gasperi había creado un Comitato nazionale per le ricerche nucleari (Cnrn) como órgano del Consejo nacional de investigaciones (CNR); a principio de los años 60 el Cnrn había sido transformado en CNEN, ente de derecho público bajo vigilancia del Ministerio de Hacienda ${ }^{25}$, y por fin en $1982^{26}$ convertido en Ente nazionale per la ricerca e lo sviluppo dell'energia nucleare e delle energie alternative (ENEA) ${ }^{27}$.

Un primer referéndum fue solicitado por un grupo de ciudadanos y declarado no admisible por la Corte constitucional con sentencia n. 31 de $1981^{28}$. La cuestión fue juzgada de modo bastante homogéneo, y afectó a un grupo de artículos de la ley de 2 de agosto de 1975, n. 393 sobre la localización de centrales nucleares y el empleo de energía eléctrica, pero resultaba inadmisible según el artículo 75 de la Constitución por estar demasiado conectada con la política energética de las Comunidades europeas, y, en particular, con la exigencia original de asegurar a través de Euratom el funcionamiento de las plantas nucleares necesarias para el desarrollo de la política energética (art. 2, let. c), con la resolución del Consejo de 17 de diciembre de 1974 que ponía entre los objetivos para 1985 el acrecentamiento de la utilización de la energía eléctrica con la evolución de la energía nuclear, con la resolución del Consejo de 13 de febrero de 1975 que aconsejaba a los Países miembros que prefiriesen la nuclear para las centrales de gran potencia, y con la resolución de 20 de noviembre de 1978, que daba indicaciones sobre los procedimientos de localización de las centrales, a pesar de la competencia de los Estados miembros. Por

14 Por ejemplo Trib. Sup. 7.1.2009; Cass. Sez. Un. 15234/2009 y 15144/2011.

15 Lo que pasó con la Provincia de Bolzano, cuya ley 10/1983 fue enviada a la Corte por el Tribunal superior con ord.362/2011, devuelta al juez a que con ord. 178/2012, en Giur. cost., 2011, 2295 por la aprobación de otra ley, de nuevo criticada por el Tribunal superior, con ord. $147 / 2012$.

16 Como en el caso de la ley 22/2011 de la Región Lombardía.

17 Por ejemplo sent.200/2012, en Giur. cost. 2012, 2910.

18 Como en la sent. 114/2012, in Giur. cost., 2012, 1648, con respeto a la ley provincial de Bolzano 7/2005, modificada con I. 4/2011.

19 L. 6 dicembre 1962, n. 1643.

20 L. 31 dicembre 1962, n. 1860.

21 L. 18 dicembre 1973, n. 856.

22 Trino vercellese (VC), Caorso (CR), Latina, Garigliano (CE).

23 Datos Terna.

24 Por la L. 2 agosto 1975, n. 393.

25 L.11 agosto 1960, n. 933; L. 15 dicembre 1971, n. 1240.

26 L.5 marzo 1982, n. 84.

27 V. por ejemplo M. Conticelli, Un'agenzia per la ricerca scientifica: l'ENEA, en Giornale dir. amm., 1999,626 ss. El ENEA ha sido varias veces reformado, una vez el 25 agosto 1991, n. 282, luego con d.lgs 30 enero 1999, n. 36, con d.lgs. 3 setiembre 2003 , n. 257 , y otra vez con I. 23 julio 2009, n. 99.

28 En Giur. Cost. 1981, 198. Un comentario en G. Gaja, Centrali nucleari: il referendum era davvero inammissibile?, Riv.dir.intern., $1981,511$. 
lo tanto, un bloque normativo de la energía nuclear hubiera entrado en conflicto con las exigencias de política exterior, en incumplimiento del art. 75.

Cuando en $1983^{29}$ el Parlamento nacional introdujo un paquete de contribuciones especiales para compensar a los municipios en los que se encuentran las plantas tecnológicamente avanzadas, destinadas al ahorro y a la recuperación de energía, a la protección del medio ambiente y a la prevención sanitaria, otro referéndum fue promovido por varios movimientos, consiguiendo más de 950.000 firmas. La Corte esta vez, pocos meses después del hecho de Chernobyl, pronunció una decisión de admisibilidad, fundada en la relevancia meramente interior de las normas impugnadas, pues las preguntas plebiscitarias no pertenecían a la política exterior ni, en particular, al Tratado de la Comunidad europea de energía atómica ${ }^{30}$ y por lo tanto no quedaban incluidas en la prohibición constitucional del art. 75 de la Constitución, sino que solamente afectaban a la localización de las centrales y a las relaciones entre municipios, Regiones, ENEL y ENEA ${ }^{31}$. Según la Corte, ni siquiera la tercera pregunta, que se refería a la capacidad de ENEL de crear sociedades anónimas participadas por entes o sociedades extranjeras con el mismo objeto, tenía alguna relación con los arts. 45 y siguientes del Tratado, que autorizaban la creación de "empresas comunes", entes comunitarios creados por el Consejo con asesoría de la Comisión, mientras que la capacidad negociadora habitual y ordinaria de ENEL puede ser discutida sin limitaciones. En pocas palabras, la Corte pasa de una interpretación extensiva del limite del art. 75 ("leyes de autorización para la ratificación de tratados internacionales") que incluye no solamente la orden de ejecución, sino también las enteras leyes de ejecución -o vinculadas con su concreta realización, incluidas las normas de actuación del derecho comunitario-, a otra, mucho más restrictiva, debido también a una formulación más analítica de las tres preguntas.

Los tres referéndums de 1987 consiguieron una participación de casi 30 millones de electores, equivalentes al $65.1 \%$ del electorado total, muy por encima del mínimo de la mayoría de los titulares del voto dispuesto por el art. 75 ; de los votantes, aproximadamente el $80 \%$ se declaró favorable a la derogación de las normas impugnadas ${ }^{32}$. El éxito de los referéndums puso el punto final a la historia de la energía atómica en Italia. El d.P.R. 500/198733 levantó acta de la derogación y desde entonces el ordenamiento italiano solo tuvo que ocuparse del desmantelamiento de las plantas existentes. De hecho, Italia fue probablemente el primer país que se enfrentó con el problema de la eliminación de las plantas nucleares cerradas, debido a la radical decisión popular de 1987. En el pasado, la solución más frecuente en el mundo era la de esperar varias décadas antes de empezar la obra de descontaminación, de desmantelamiento y de gestión de los residuos, para favorecer con el paso del tiempo la degradación natural de la mayor parte de la contaminación existente: ese período de espera se suele definir como la custodia tutelar pasiva o "safe store". En el caso italiano, los recursos financieros necesarios tenían que ser facilitados a través de la disposición por el monopolista público de un porcentaje del precio de cada kWh producido. Las cuatro centrales existentes, una de las cuales ya fue cerrada en 1982, fueron puestas en "safe store", como las nuevas centrales de Montalto di Castro y Trino 2, que se estaban construyendo.

Pero en 1999, cuando la liberalización del sector eléctrico implicó la privatización de ENEL y su listado de bolsa, fue fundada una sociedad anónima (Sogin), de propiedad del Tesoro, para el desmantelamiento inmediato de las centrales, a las que se sumaron en 2003 las plantas de investigación de ENEA y en 2005 la de fabricación de combustible de Bosco Marengo (AL), y de las de Saluggia (VC), Casaccia (Roma) y Rotondella (MT). Una de las plantas, proyectada por famosos arquitectos y construida hacía más de cincuenta años, fue mientras tanto declarada de interés monumental y como tal protegida, de manera que forma objeto de dos procedimientos administrativos, uno de conservación y el otro de desmantelamiento. Todo el material radioactivo ha sido reprocesado en el extranjero, después de algunas pruebas iniciales de almacenamiento en seco ${ }^{34}$.

Después de más de veinte años desde el referéndum de 1987 el Parlamento italiano ha reexaminado la materia con una nueva intervención normativa. El decreto-ley 112/2008, convertido con modificaciones en ley 133/200835, la ley 99/2009 $9^{36}$ y los decretos legislativos 31/2010 y 104/201037 reintrodujeron procedimientos de localización de

29 L. 10 enero 1983, n. 8, art. unico.

30 Ratificado por Italia con I. 14 octubre 1957, n. 1203.

31 N. 25 de 16 de enero-3 de febrero de 1987, en Giur. Cost., 1987, 141 ss.

32 De 45.870 .000 electores, entre 29.855 .604 y 29.871 .570 se pronunciaron para la abrogación de las tres leyes.

33 De 9 de diciembre.

34 Cfr. ARPA Rivista, n.5, 2009, 16 ss. V. P.M. Putti, “Il decommissioning” degli impianti nucleari”, en Annuario di diritto dell'energia, Bolonia, 2011, 188 ss.

35 L. 6 agosto 2008, n. 133, para la definición de la política energética nacional, incluida la realización de plantas de producción de energía nuclear.

36 L. 23 julio 2009, n. 99, de delegación al Gobierno para la localización de plantas de fabricación, de sistemas de almacenamiento de combustible y depósito de rechazos radioactivos y de medidas compensativas.

37 D.lgs. 15 febrero 2010, n. 31 y d.lgs. 2 julio 2010, n. 104. 
centrales nucleares y formas de producción de energía atómica en el territorio nacional ${ }^{3}$. La Corte esta vez declaró admisible el referéndum de revocación de esas normas, con sentencia 28/201139. El límite del Tratado Euratom parece ahora no implicar ningún vínculo: el Tratado sólo impone obligaciones de investigación y seguridad, pero no obliga a cada Estado a incluir la energía nuclear en sus programas energéticos ni excluye que prohíba plantas nucleares en su territorio, estableciendo su propio mix energético según políticas autónomas. Para que el límite del art. 75 se aplique no es suficiente que la pregunta plebiscitaria se refiera a una materia que haya sido objeto de tratados internacionales; hace falta que entre en conflicto con específicas obligaciones derivadas de los tratados, de manera que pueda surgir una responsabilidad internacional para el Estado.

El referéndum del 12 y 13 de junio de 2011 consiguió de nuevo un resultado muy desfavorable a cualquier posibilidad de cualquier instalación nuclear en Italia. Con una afluencia de electores de aproximadamente el 57,01 o del $54,79 \%$ considerando los residentes en el extranjero, el 94,05\% contestó sí a la anulación.

\section{ENERGÍA EÓLICA}

En cuanto a la energía eólica, el legislador nacional, después de haber aplicado la normativa europea para favorecer su introducción ${ }^{40}$, ha repartido los objetivos de producción entre Regiones ("burden sharing")"41. Muchas Regiones, y también administraciones locales, han tratado de crear obstáculos a la expansión de las plantas eólicas con técnicas varias, argumentando que su excesiva difusión implica un perjuicio al paisaje, comprometiendo valores estéticos, históricos y culturales, y al final causando también pérdidas a nivel económico por la depreciación del patrimonio explotable turisticamente. Por lo tanto, el Parlamento ha impuesto la adopción de líneas directivas para la articulación del procedimiento para la autorización a construir y explotar parques eólicos, con el propósito de garantizar la correcta inserción de las instalaciones eólicas en el paisaje, combinando las exigencias de simplificación, típicamente traducidas en el permiso único destinado a sustituir cualquier título de autorización, con la necesidad de protección ambiental ${ }^{42}$. La Conferencia unificada Estado-Regiones-Autonomías locales se pronunció sobre las directivas, para conseguir el máximo nivel de consenso, y quedó firme el resultado en términos de incremento mínimo de energía producida de fuentes renovables ${ }^{43}$. Finalmente aprobadas con decreto ministerial del Ministro de Hacienda de acuerdo con el de Medio ambiente del 10 de setiembre de 2010, las directivas han sido impugnadas en Corte constitucional por la Provincia autónoma de Trento por conflicto de atribuciones. El recurso fue declarado fundado con sentencia $275 / 2011^{44}$, por vulnerar la competencia provincial en materia de paisaje, por cuanto el decreto definía con detalle los criterios para la decisión de admisibilidad de aprobación de la instalación de los parques eólicos.

Sin embargo, la tensión entre diferentes niveles de gobierno no presenta indicios de suavizarse. La jurisprudencia de mérito sigue ocupándose de casos en los que las comunidades locales han hecho esfuerzos para bloquear nuevos parques, hasta haber infringido los principios fundamentales del procedimiento, sobre todo en las Regiones del Sur, que presentan condiciones morfológicas y climáticas más favorables al uso de viento con fines de producción de energía. Denegaciones sin adecuada motivación, pretextos, repetición innecesaria de fases del procedimiento ya finalizadas, silencio... son todavía frecuentes ${ }^{45}$. En otros casos, los juzgados administrativos tuvieron que anular convenios en las que las empresas privadas fueron obligadas a aceptar condiciones económicas muy desfavorables, como contribuciones compensatorias al daño paisajístico o social, a pesar de la clara prohibición del d.lgs. 387 (art. 12, aptd. 6) ${ }^{46}$.

La Corte constitucional tuvo que declarar ilegítimas algunas leyes regionales que perseguían el mismo rumbo, como la de Cerdeña que consentía a los Ayuntamientos indicar las áreas industriales, limítrofes a las industriales, o ambientalmente comprometidas como idóneas para albergar parques eólicos, invirtiendo el criterio estatal, que prescribe la individualización regional de las áreas inidóneas, y prohíbe ubicar parques eólicos en áreas agrícolas, con otra inversión del criterio básico ${ }^{47}$. En varias otras decisiones, la Corte ha precisado los límites entre las competencias estatales y las regionales en esta materia ${ }^{48}$.

38 V. L. Ammannati, M. de Focatiis, Un nuovo diritto per il nucleare. Una prima lettura del d.lgs. n.31/2010, www.astrid-online.it; G. Morbidelli, “La procedura di localizzazione degli impianti”, en Annuario dell'energia, cit., 121 ss.

39 En Giur. cost.2011, 333; v. también sent.22 julio 2010, n.278, in Giur. cost., 2010, 3241. V. M. D’Alberti, “La localizzazione degli impianti nucleari", en Annuario di diritto dell'energia, cit., 168.

40 Dir 77/2001/CE y dir.28/2009/CE.

41 D.lgs. 24 diciembre 2007, n. 244, art. 2, aptd. 167, modificado por la l. 27 febrero 2009, n. 13 , art. 8 bis.

42 D.lgs. 29 diciembre 2003, n. 387, art. 12, aptd. 10.

43 D. Igs. 244/2007, art. 2, aptd. 167.

44 En Giur. cost. 2011, 3563.

45 V. por ejemplo Tar Puglia, Lecce, sec. I, 821/2012; Tar Calabria, Catanzaro, sec. I, 676/2012, 728 /2012, 729/2012 y 732/2012.

46 Por ejemplo Tar Puglia, Lecce, sec.l, 1361/2013 y 1347/2013.

47 Sent. 224/2012, in Giur cost. 2012, 3363.

48 Sent. 168/2010, 275/2011, 80/2013, in Giur. cost. 2010, 2028; 2011, 3563 y 2013, 1330.

REALA, n 2 julio-diciembre 2014, ISSN: 1989-8975 - DOI: http://dx.doi.org/10.24965/reala.v0i2.10191 
A pesar de la resistencia activa y pasiva de muchas autoridades locales y regionales, la demanda de producción de energía eólica ha crecido un 20\% entre 2010 y 2011 y un 34\% entre 2011 y 2012, llegando a una facturación superior a los 2 mil millones de Euros.

\section{SECTOR FOTOVOLTAICO}

La misma tensión entre autoridades locales y normas estatales condicionadas por directivas europeas ${ }^{49}$ se ha manifestado en el sector de la energía fotovoltaica. Aquí también muchas Regiones, no solamente del Sur, han impuesto con ley moratorias o suspensiones de extensas áreas de su territorio, consideradas incompatibles con parques fotovoltaicos por la calidad de su valor ambiental o paisajístico. La Corte constitucional ha sancionado esas pretensiones con varias sentencias ${ }^{50}$, asumiendo que las exigencias de protección del paisaje deben retroceder frente a los principios de origen comunitario, como en el caso de la Región Basilicata, que había imposibilitado cualquier planta fotovoltaica, pero admitía excepciones en presencia de condiciones muy restrictivas ${ }^{51}$.

\section{BIOENERGÍAS}

La fuente principal de bioenergía en Italia está representada por las biomasas, o sea por los combustibles sólidos de origen orgánico. De hecho, más o menos la mitad de la producción eléctrica a partir de biomasas sólidas deriva de la combustión de residuos sólidos urbanos ${ }^{52}$.

Aun cuando la materia de la energía a partir de los residuos fuese ya conocida desde el decreto "Ronchi" 53 y el d.lgs. 112/199854, que en el momento de devolver a las Regiones las competencias en materia de plantas productivas de energías de fuentes renovables hacía expresa referencia a las plantas que producen energía a partir de residuos ${ }^{55}$, fue la directiva $77 / 2001 /$ CE, desarrollada por el d.Igs. 387/2003, la que introdujo el concepto de biomasa, identificando su característica básica en la directa interferencia con el régimen de gestión de residuos.

\section{ENERGÍAS GEOTÉRMICAS}

La directiva define la biomasa como la parte biodegradable de los productos, desechos o residuos procedentes de agricultura, incluidas las substancias vegetales y animales, de silvicultura y de industrias relacionadas, y también las partes biodegradables de los residuos industriales y urbanos ${ }^{56}$. Por lo tanto, la directiva, en los considerandos introductorios, evidencia la preocupación que la política de incentivación a la producción energética de biomasas no perjudique la política también comunitaria de gestión de los residuos. De la misma manera, en Italia se ha creado una tensión entre la regulación de las renovables (d.Igs. 387/2003) y la de los residuos (d.lgs. 152/2006), que los jueces administrativos han resuelto poniendo énfasis sobre el concepto "subjetivo" de residuo: como el valor de los materiales no es intrínseco, sino que depende de la valoración de los que los poseen, no existe una sustancia que pueda ser definida generalmente y en cualquier caso como residuo, sin perjuicio de su destino al reciclado y en manera especial a la producción de energía ${ }^{57}$. La averiguación de este dato determina la aplicación de la regulación favorable a las renovables. La cuestión no cambia después de la dir.2009/28/CE y de su d.lgs. de aplicación 28/2011, que en la definición de biomasas incluyen ahora también la fracción biodegradable de productos, residuos o desechos de siega y poda desde verde público o privado. Como siempre, muchos litigios provienen de la oposición a la construcción de termovalorizadores por parte de ciudadanos residentes en el territorio colindante, directamente o a través de asociaciones medioambientales ${ }^{58}$, y de los entes territoriales representativos de sus comunidades, mediante el ejercicio de los poderes de gestión del territorio y de las actividades constructivas ${ }^{59}$. Varias Regiones han intentado también introducir obstáculos con disciplinas declaradas inconstitucionales por la Corte $^{60}$. Otras leyes regionales ${ }^{61}$ orientadas a la simplificación del procedimiento para el otorgamiento del título de edificación han sobrevivido al juicio de la Corte, en cuanto respetuosas en el reparto de competencias entre Estado y Regiones en las materias concurrentes de energía y gobierno del territorio y en la exclusiva y

49 Como las dir. 2001/77/CE y 2009/28/CE.

50 Sent. 85/20112, en Giur. cost. 2012, 1180; sent. 192/2011, ibídem, 2481, que se refieren respectivamente a la I. 7/2011 de la Región Veneto y a la l. 18/2010 de la Región de Piemonte.

51 Sent. 67/2011, en Giur. cost., 2011, 1025, respecto a la I. 42/2009.

52 Rapporto GSE 2011.

53 Artt. 4, 8, 22, 31 d.lgs. 5 febbraio 1997, n. 22.

54 Art. 30.

55 Art. 29, let. g.

56 Art. 2, let. d, dir. 77/2001/CE y art. 2, d.Igs. 29 diciembre 2003, n. 387. Muy parecida la definición de la dir. 2009/28/CE.

57 V. por ejemplo Tar Piemonte, Sec. I, sent. 1563/2009.

58 Por ejemplo Tar Piemonte, Sec.I, 1340/2011 y 635/2011.

59 Por ejemplo, Tar Campania, Napoli, Sec.II, 3014/2013 y Tar Calabria, Catanzaro, 1656/2011.

60 Como la Región Marche, con ley 31/2009: v. sent. 332/2010, en Giur. cost. 2010, 4766.

61 Como la ley Toscana 71/2009, art. 10, apt. 2: v. sent. 313/2010, en Giur. cost. 2010, 4392. 
trasversal de la protección del medio ambiente, y también de los principios de legislador estatal, que se inclina favorablemente hacia las renovables. En los procesos constitucionales muchas veces particulares o asociaciones medioambientales intervienen ad opponendum o ad adiuvandum, y tradicionalmente la Corte los declara carentes de legitimación ${ }^{62}$. Esta clase de energía, aunque sea renovable, encuentra bastante oposición popular, debido a la posible contaminación por las emisiones y a la lamentable disminución de residuos de recogida diferenciada ${ }^{63}$. Hasta ahora Italia ha conseguido reciclar solamente el 31\% de los residuos, frente al objetivo europeo de $65 \%$ en 2020 , y creciendo la cantidad de residuos reciclados, disminuye el combustible que pueda llegar a los termovalorizadores.

7. La fuente normativa de los recursos geotérmicos es la antigua ley de minas, el r.d. 1443/1927. Todavía se encuentran allílos procedimientos para las concesiones del uso de los recursos geotérmicos ${ }^{64}$. La crisis petrolífera de 1973-74 ha dado impulso a la búsqueda y a la explotación de hidrocarburos, pero también de recursos geotérmicos. Por lo tanto, en los años 80 la ley-marco de la geotermia 896/1986 ha adoptado una nueva reglamentación de la materia, disciplinando principios generales, procedimientos de otorgamiento de permisos, obligaciones del concesionario, etc. La ya citada ley $387 / 2003^{65}$, listando las energías renovables que aprovechan el régimen especial de permiso único -que en caso de necesidad vale para la modificación del instrumento urbanístico-, menciona expresamente los recursos geotérmicos.

El d.lgs. $22 / 2010^{66}$ ha operado una revisión de la ley-marco, adecuándola e integrándola de acuerdo con los principios europeos introducidos por la dir. 79/2009, como la liberalización del mercado eléctrico, las características mineras de la geotermia, y en general las políticas de promoción de las energías renovables y de eficiencia energética, a parte del nuevo equilibrio de competencias entre Estado, Regiones y entes locales después de la revisión constitucional de 2001. El texto único, de hecho, concentraba en el Estado todas las funciones sobre la materia, siendo los recursos geotérmicos patrimonio indisponible del Estado según el art. 826 del código civil de 1942. Ya el d.lgs 112/1998 (Ilamado "Bassanini") había delegado a las Regiones las funciones de otorgamiento de permisos de investigación y explotación en tierra. Por lo tanto había necesidad de una devolución de competencias constitucionalmente adecuada.

La Corte constitucional se ha pronunciado en varias sentencias sobre la legitimidad de leyes regionales impugnadas por el Estado, a veces por incumplimiento de principios estatales por detalles acerca de la investigación de recursos geotérmicos y construcción de plantas ${ }^{67}$ o de introducción de prohibiciones generales de localización de plantas en áreas de valor paisajístico o medioambiental ${ }^{68}$. La Corte ha pronunciado varias sentencias de inadmisibilidad, y nunca ha llegado al fondo de la cuestión, relativo a la constitucionalidad de las disposiciones impugnadas ${ }^{69}$. Recíprocamente, la regulación estatal ha resistido a las impugnaciones regionales ${ }^{70}$. El ulterior

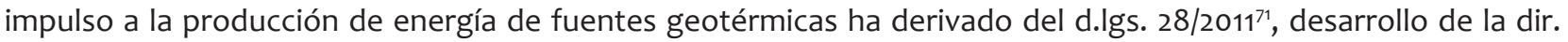
2009/28/CE, dedicado a la promoción de investigaciones y desarrollo de plantas geotérmicas de reducido impacto medioambiental, centrales de impacto cero y centrales a medio-baja potencia.

El parque italiano de plantas geotermoeléctricas ha crecido en términos constantes desde 1997 y hasta 2008, con un índice medio de 0,9\% por año en cuanto a número, 2,2\% en cuanto a potencia, 3,2\% en cuanto a producción. El $67,7 \%$ de las plantas (21 de 31) pertenece a la clase de potencia entre 10 y $20 \mathrm{MW}$. El 12,9\% está incluso en la clase de las plantas pequeñas, por debajo de los $10 \mathrm{MW}$. Todas las plantas italianas están localizadas en Toscana. Italia ocupa la tercera posición en el mundo en cuanto a plantas geotérmicas, después de Estados Unidos y México.

62 Por ejemplo, sent. 254/2009, 246/2009, 250/2009, 233/2009.

63 Las plantas de este tipo eran 71 en 2010 y el número no ha subido en los dos años después, con un ligero incremento de producción: datos GSE.

64 Art. 1.

65 Art. 2, apt. 1, let. a.

66 D.Igs. 11 febbraio 2010, en actuación de la ley de delegación 23 luglio 2009, n.99.

67 Por ejemplo L. Provincia Bolzano 15/2011, art. 25 o L. Campania 8/2008, artt.33 y 45.

68 Como en el caso de la ley Friuli-V.G. 6/2011, art. 1.

69 Sent. 114/2012 acerca de la ley de Bolzano, en Giur. cost., 2012, 1648; 1/2010 en respecto de la ley de Campania, en Giur. cost. 2012, 1397; 100/2012, en Giur. cost., 2010, 3397.

70 Sent. 112/2011, en Giur. cost. 2011, 1509 y 278/2010, en Giur. cost., 2010, 3397

71 De 3 mayo. 